\title{
Novel MLH1 duplication identified in Colombian families with Lynch syndrome
}

\author{
Virginia Alonso-Espinaco, $P h D^{1}$, María Dolores Giráldez, $M D^{1}$, Carlos Trujillo, $M D, P h D^{2}$, \\ Heleen van der Klift, PhD ${ }^{3}$, Jenifer Muñoz, MSc ${ }^{l}$, Francesc Balaguer, $M D$, PhD', Teresa Ocaña, $M S c^{l}$, \\ Irene Madrigal, $P h D^{4}$, Angela M. Jones, $M S c^{5}$, M. Magdalena Echeverry, $P h D^{6}$, \\ Alejandro Velez, MD, PhD ${ }^{7}$, Ian Tomlinson, $M D$, PhD ${ }^{5}$, Montserrat Milà, $P h D^{4}$, Juul Wijnen, $P h D^{3}$, \\ Luis Carvajal-Carmona, PhD ${ }^{5,6}$, Antoni Castells, $M D, P h D^{I}$, and Sergi Castellví-Bel, $P h D^{l}$
}

\begin{abstract}
Purpose: Lynch syndrome accounts for $2-4 \%$ of all colorectal cancer, and is mainly caused by germline mutations in the DNA mismatch repair genes. Our aim was to characterize the genetic mutation responsible for Lynch syndrome in an extensive Colombian family and to study its prevalence in Antioquia. Methods: A Lynch syndrome family fulfilling Amsterdam criteria II was studied by immunohistochemistry and by multiplex ligation-dependent probe amplification (MLPA). Results were confirmed by additional independent MLPA, Southern blotting, and sequencing. Results: Index case tumor immunohistochemistry results were MLH1-, MSH2+, MSH6+, and PMS2-. MLPA analysis detected a duplication of exons 12 and 13 of MLH1. This mutation was confirmed and characterized precisely to span 4219 base pairs. Duplication screening in this family led to the identification of six additional carriers and 13 noncarriers. We also screened 123 early-onset independent colorectal cancer cases from the same area and identified an additional unrelated carrier. Conclusion: A novel duplication of exons 12 and 13 of the MLH1 gene was detected in two independent Lynch syndrome families from Colombia. A putative founder effect and prescreening Lynch syndrome Antioquia families for this specific mutation before thorough mismatch repair mutational screening could be suggested. Genet Med 2011:13(2):155-160.
\end{abstract}

Key Words: Lynch syndrome, HNPCC, MMR genes, hereditary cancer, Colombia

Co olorectal cancer (CRC) is the second leading cause of cancer death in the developed world, whereas in developing countries this neoplasm represents the sixth or seventh cause of cancer death. Lynch syndrome (LS), an autosomal dominant hereditary disorder, is the most commonly inherited form of CRC and accounts for $2-4 \%$ of all CRC. ${ }^{1}$ In Colombia, CRC is

From the ${ }^{1}$ Gastroenterology Department, Institut de Malalties Digestives i Metabòliques, Hospital Clínic, CIBEREHD, IDIBAPS, University of Barcelona, Barcelona, Catalonia, Spain; ${ }^{2}$ Genetics Laboratory, Dr. Erfan \& Bagedo General Hospital, Jeddah, Saudi Arabia; ${ }^{3}$ Center for Human and Clinical Genetics, Leiden University Medical Center, Leiden, The Netherlands; ${ }^{4}$ Biochemistry and Molecular Genetics Department, Hospital Clínic, CIBERER, IDIBAPS, Barcelona, Catalonia, Spain; ${ }^{5}$ Wellcome Trust Centre for Human Genetics, Nuffield Department of Medicine, University of Oxford, Oxford, United Kingdom; ${ }^{6}$ Department of Biology, University of Tolima, Ibague, Colombia; and ${ }^{7}$ Department of Pathology, Pablo Tobon Uribe Hospital, Medellin, Colombia.

Sergi Castellví-Bel, PhD, Gastroenterology Department, Hospital Clinic, Villarroel 170, Barcelona 08036, Spain. E-mail: sbel@clinic.ub.es.

Disclosure: The authors declare no conflict of interest.

Submitted for publication August 11, 2010.

Accepted for publication October 21, 2010.

Published online ahead of print January 12, 2011.

DOI: $10.1097 / G I M .0 b 013 \mathrm{e} 318202 \mathrm{e} 10 \mathrm{~b}$ the fourth cause of cancer mortality, and it is estimated that approximately 2400 individuals die annually due to this cancer, ${ }^{2}$ and approximately 75 persons die annually due to LS. ${ }^{3}$

LS is characterized by high penetrance, early-onset CRC and an increased risk for extracolonic tumors (endometrium, stomach, small bowel, ovary, hepatobiliary tract, renal pelvis, and ureter). Clinical diagnosis of LS based on the Amsterdam II criteria or revised Bethesda guidelines can be difficult because family history information is often incomplete, and a typical clinical phenotype such as diffuse polyposis is not present. ${ }^{4,5}$ However, its early recognition is essential to identify high-risk individuals who will require intensive cancer surveillance. A significant patient survival advantage and reduction in the incidence of colorectal tumors have been observed after colonoscopic screening and polypectomy. ${ }^{6}$

Germline mutations in DNA mismatch repair (MMR) genes, most commonly $\mathrm{MLH1}$ and $\mathrm{MSH} 2(90 \%)$, less frequently $\mathrm{MSH} 6$ $(10 \%)$, and rarely PMS2 and EPCAM, are responsible for the majority of cases of LS. ${ }^{1}$ However, MSH6 and PMS2 are less studied in molecular LS screenings, and mutations in these genes could be largely underestimated (van der Klift et al., unpublished study). Mutations in MMR genes impair the function of the corresponding MMR protein, which normally recognize and repair mismatched nucleotides caused by DNA polymerase slippage mispairing. This MMR alteration results also in absence of the corresponding protein and microsatellite instability in the tumor tissue. Therefore, microsatellite instability analysis and tumor immunohistochemistry (IHC) can provide a comprehensive picture of molecular abnormality in patients with LS by demonstrating the MMR alteration and pinpointing the specific gene most likely to be mutated.

Recently, additional screening for genomic rearrangements in the MMR genes by multiplex ligation-dependent probe amplification (MLPA) has also been added to the LS genetic testing routine because these molecular defects have been found to be also causative of LS, and they are not detected by the aforementioned screening techniques. ${ }^{7-10}$

So far, there is only one LS study including molecular screening in the Colombian population. ${ }^{10}$ That study included 23 unrelated Colombian families with LS that were screened for point mutations in the $M L H 1$ and $M S H 2$ genes with a mutation detection rate of $48 \%$. However, MLPA was not performed previously to the thorough exon scanning by single strand conformation polymorphism. Therefore, some of the patients with LS in that previous study without a mutation in the MMR genes may have some undetected genomic rearrangements in their MMR repair genes.

The aim of our study was to identify a pathogenic germline mutation in an extensive Colombian family fulfilling Amsterdam II criteria to give genetic counseling and provide a presymptomatic and confirmatory molecular diagnosis, and 


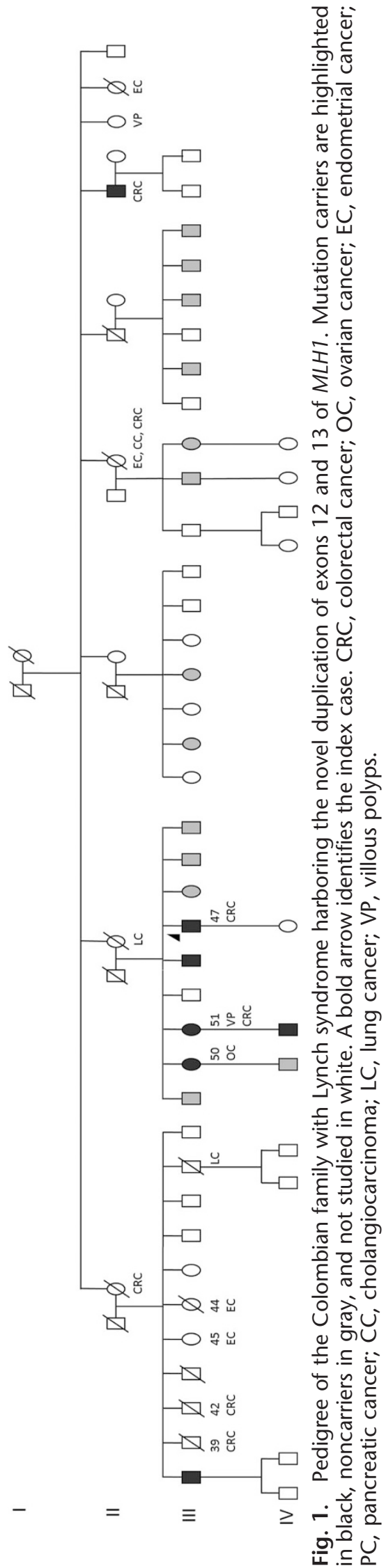

to study its prevalence in this population. We have identified a novel duplication of exons 12 and 13 of the $M L H 1$ gene by MLPA, DNA quantitative polymerase chain reaction (PCR), Southern blotting, and sequencing. This mutation was also detected in an unrelated LS family from the same Colombian province, suggesting a putative founder effect in this population.

\section{MATERIALS AND METHODS}

\section{Patients}

We studied an extensive family that fulfilled Amsterdam II criteria, ${ }^{4}$ and were from the Antioquia province in central Northwestern Colombia. ${ }^{4}$ A family tree depicts 66 members in four generations and six family nuclei (Fig. 1).

Individuals from this family developed CRC more commonly, although other neoplasms were also present including endometrial cancer, pancreatic cancer, ovarian cancer, lung cancer, and cholangiocarcinoma. One of their members (II-8) initially presented with a CRC at age 49 years, then endometrial cancer at age 54 years, and later cholangiocarcinoma at age 64 years. Villous polyps were also found in two members of this family (in one case without concurrent CRC). Personal and familial history data were reported by a family member. Individual cancer diagnosis and onset age were confirmed by reviewing medical reports. An additional Colombian cohort (123 early-onset unrelated CRC cases and 80 cancer-free controls) was also available.

Informed consent to perform the molecular analysis was approved by the Hospital Clínic of Barcelona, the University of Tolima, and Pablo Tobon Uribe Hospital and was obtained from all individuals who participated in this study.

\section{DNA and tissue samples}

DNA samples extracted from peripheral blood were available from 20 individuals of this family. DNA extraction was performed in reference centers in Colombia and Miami, FL. The index case (III-17) was studied initially, and afterward, when the LS mutation was identified, mutational screening was offered to the rest of the family. Up to now, 19 additional members have been screened for this specific mutation. IHC data for MMR proteins were available from CRC tumor lesions of two members of this family (index case and III-14). IHC analyses were performed in the Department of Pathology of the Ullevål University Hospital (Oslo, Norway). DNA samples from an additional Colombian cohort (123 early-onset unrelated CRC cases and 80 cancer-free controls) were screened for the presence of the mutation identified in this study.

\section{MMR mutational screening: MLPA}

Mutational screening in the MMR genes was initiated, as recommended, by performing MLPA before extensive exon scanning to detect genomic rearrangements. We used a test for the MLH1/MSH2 genes available commercially (P003MLH1/MSH2, MRC-Holland, Amsterdam, The Netherlands; www.mrc-holland.com) according to manufacturer's instructions. Samples were loaded onto a 3130 Genetic Analyzer (Applied Biosystems, Foster City, CA), and results were visualized using the Gene Mapper program and analyzed with the SEQUENCE Pilot-module MLPA $^{\circledR}$ program (JSI medical systems GMBH, Kippenheim, Germany). 


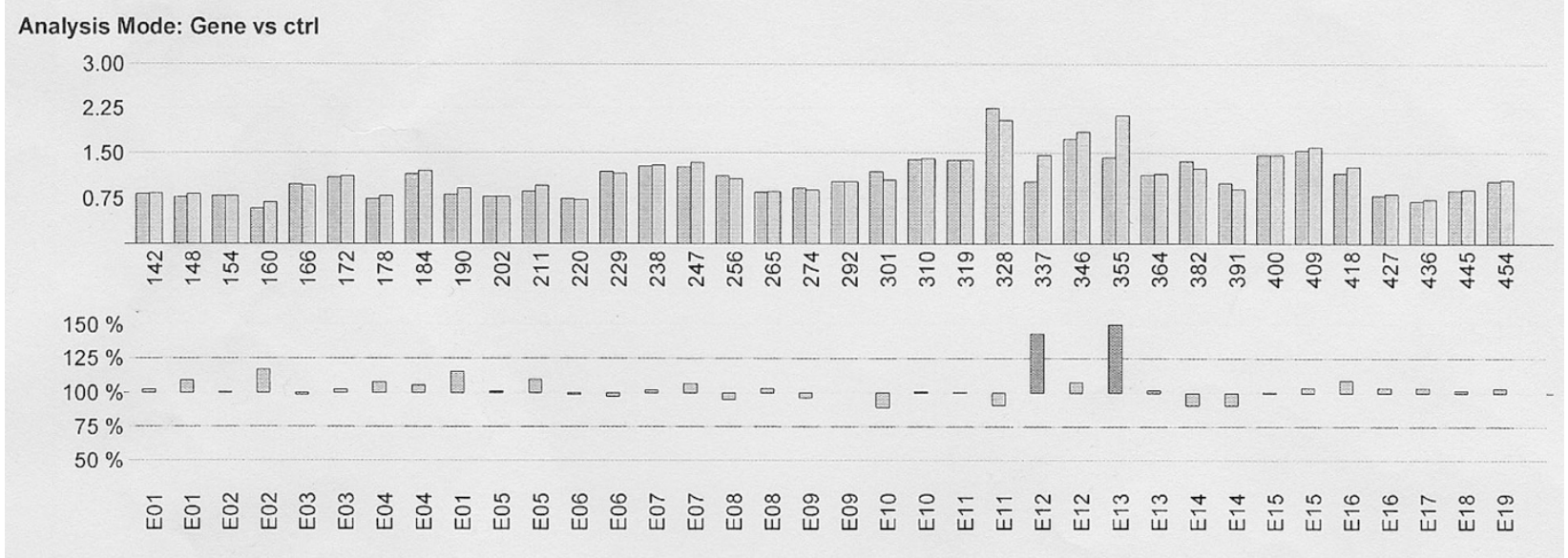

Fig. 2. MLPA test showing the duplication in exons 12 and 13 of the $M L H 1$ gene in the index case using the SEQUENCE Pilot-module MLPA ${ }^{\circledR}$ program. Upper diagram, Amplification sizes corresponding to individual MLH1/MSH2 exons are displayed horizontally (a complete specification can be found at http://www.mrc-holland.com). In the vertical axis and displayed side-to-side, dark shadow boxes correspond to relative peak area (RPA) in control DNA and light shadow boxes to RPA in the analyzed patient DNA. Lower diagram, Patient DNA/control DNA ratio RPA is displayed for individual MLH1/MSH2 exons. Patient DNA/control DNA ratio RPA for MLH1 exons 12 and 13 in the index case was $135 \%$ higher as highlighted by the corresponding two dark shadow boxes, suggesting an heterozygous duplication of these exons.

\section{Confirmation of MLPA results: Additional MLPA and quantitative DNA real-time PCR}

Results with the P003-MLH1/MSH2 MLPA kit were confirmed by also using the MLPA P248-MLH1-MSH2 kit (MRCHolland, Amsterdam, The Netherlands). This MLPA kit is directed to the same genes and exons as the P003-MLH1/MSH2 MLPA, but probe sites are located independently.

Additionally, to confirm the duplication of exons 12 and 13 of the $M L H 1$ gene, a quantitative DNA real-time PCR was also performed. The index case and two control DNAs were analyzed in triplicate for exons $11,12,13$, and 14 of $M L H 1$ and for exon 8 of an unrelated gene (PARVG). Eight microliters of each DNA sample (at $0.1 \mathrm{ng} / \mu \mathrm{L}$ ) was mixed with SYBR Green PCR Master Mix and $20 \mathrm{pmol}$ of each primer to a final volume 25 $\mu \mathrm{L}$. PCR conditions in a 7300 Real-Time PCR System (Applied Biosystems, Foster City, CA) were $95^{\circ} \mathrm{C}$ for 10 minutes; 40 cycles at $95^{\circ} \mathrm{C}$ for 15 seconds and $58^{\circ} \mathrm{C}$ for 1 minute; and a final dissociation stage of $95^{\circ} \mathrm{C}$ for 15 seconds, $60^{\circ} \mathrm{C}$ for 1 minute, $95^{\circ} \mathrm{C}$ for 15 seconds, and $60^{\circ} \mathrm{C}$ for 15 seconds. Amounts of DNA in each amplification were determined by comparing the results to a standard curve produced by real-time PCR of serial dilutions (e.g., undiluted, 1:4, 1:16, and 1:64) of a known amount of DNA.

\section{Molecular characterization of the $M L H 1$ duplication: Southern blotting and sequencing}

Southern blot analysis was performed to get information about the size of the duplication and the location of the breakpoints. HindIII, ScaI, and Bsp1407I digests of genomic DNA of the index patient were hybridized with a specific cDNA probe encompassing exons 11-19 of MLH1. Analysis of the aberrant restriction patterns combined with an in silico restriction map of the genomic region of interest (Webcutter2.0; www.rna.lundberg.gu.SE/cutter2/) enabled us to identify a region where breakpoints will be most likely located. In that region, a duplication-specific PCR was designed with a

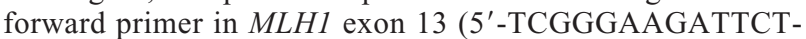
GATGTGG-3') and a reverse primer in exon 12 (5'-GAC-
CTTATCACTACTTCCAG-3'), predicted to amplify a 1-1.5 $\mathrm{kb}$ PCR product from genomic DNA of the patient. This PCR product was used to sequence across the breakpoints with an internal forward primer (5'-GTTACAGAGCAAGGAGTTGA-3') and the same reverse PCR primer.

\section{Specific PCR amplification for $M L H 1$ duplication screening}

To rapidly screen for the MLH1 duplication in other members of this family or in independent Colombian CRC families, a specific PCR amplification was performed combining a new set of primers MLH1-12aR (5'-GAATCTGTACGAACCATCTG-3') and MLH1-13F (5'-TGCAACCCACAAAATTTGGC-3'). These primers were combined with other exon 12 amplification primers (12aF: 5'-ATTTGGGGACCTGTATATCT-3' and 12cR: 5'-ACAGAATAAAGGAGGTAGGC$\left.3^{\prime}\right)$ to disregard lack of PCR amplification due to low-quality DNA. Then, if DNA is not degraded, noncarriers and duplication carriers will show 233 and 513 base pair (bp) fragments, whereas a 1289-bp band will only be present in duplication carriers (Fig. 3B). Cycling conditions were $94^{\circ} \mathrm{C}$ for 1 minute; 35 cycles at $94^{\circ} \mathrm{C}$ for 15 seconds, $53^{\circ} \mathrm{C}$ for 15 seconds, and $72^{\circ} \mathrm{C}$ for 30 seconds; and a final extension of $72^{\circ} \mathrm{C}$ for 3 minutes.

\section{RESULTS}

A large Colombian family fulfilling clinical Amsterdam II criteria was referred to our laboratory for LS molecular testing (Fig. 1). IHC data for tumor MMR proteins was available for the index case (III-17) and an additional sibling (III-14), being MLH1 - , MSH2+, MSH6+, PMS2-, compatible with a genetic defect in the MLH1 gene. DNA from the index case affected with CRC at age 47 years (male, III-17) was sent for molecular MMR testing.

Mutational screening in the MMR genes was initiated, as recommended, by performing MLPA before extensive exon scanning to detect possible genomic rearrangements. When we 
used a test for the MLH1/MSH2 genes, we were able to detect a heterozygous duplication apparently involving exons 12 and 13 of the MLH1 gene (Fig. 2). Afterward, to confirm this duplication by other independent molecular techniques, we performed an additional MLPA test and a DNA quantitative realtime PCR. The additional MLPA test was directed to the same genes and exons as the P003-MLH1/MSH2 MLPA, but probe sites were located independently. This additional MLPA test confirmed that exons 12 and 13 of $M L H 1$ were duplicated in the index case DNA (results not shown). Additionally, this duplication was also confirmed by performing a quantitative DNA real-time PCR (results not shown). Both independent techniques confirmed this duplication and showed that the alteration was only restricted to exons 12 and 13, and it did not extend to exons 11 or 14 of the MLH1 gene.

To further characterize this novel duplication at the molecular level, Southern blotting and sequencing were performed on the index case DNA. Southern blot analysis consistently showed an aberrant fragment of $4-4.2 \mathrm{~kb}$, and sequencing across the break- points permitted determination of the exact size of the duplication: 4219 bp. Duplication breakpoints are lying in long T stretches, a $(\mathrm{T})_{21}$ located 8 bp upstream of exon 12 and a $(\mathrm{T})_{19}$ in intron 13 starting 896 bp downstream of exon 13 (Fig. 3). This duplication should be described as c.1039-8T_1558 + 896Tdup following mutation nomenclature recommendations (GenBank accession: NM_000249; http://www.hgvs.org/mutnomen/). ${ }^{11}$

Moreover, to screen for the $M L H 1$ duplication in additional unrelated Colombian CRC cases, a rapid specific PCR amplification was used combining existing primers for exons 12 and 13. PCR amplification using primers $13 \mathrm{~F}$ and $12 \mathrm{aR}$ was only able to yield a fragment of $1289 \mathrm{bp}$ in exons 12 and $13 \mathrm{MLH1}$ duplication carriers, as it is depicted in Figure 3.

Once this novel $M L H 1$ duplication was thoroughly confirmed to be the causative LS mutation in the index case DNA, we proceeded to offer molecular diagnostic, either confirmatory or presymptomatic, to the rest of his family. By doing so, we were able to identify this $\mathrm{MLH1}$ duplication in heterozygous form in 6 of the 19 additional studied family members (Fig. 1).

\section{A MLH1 gene}
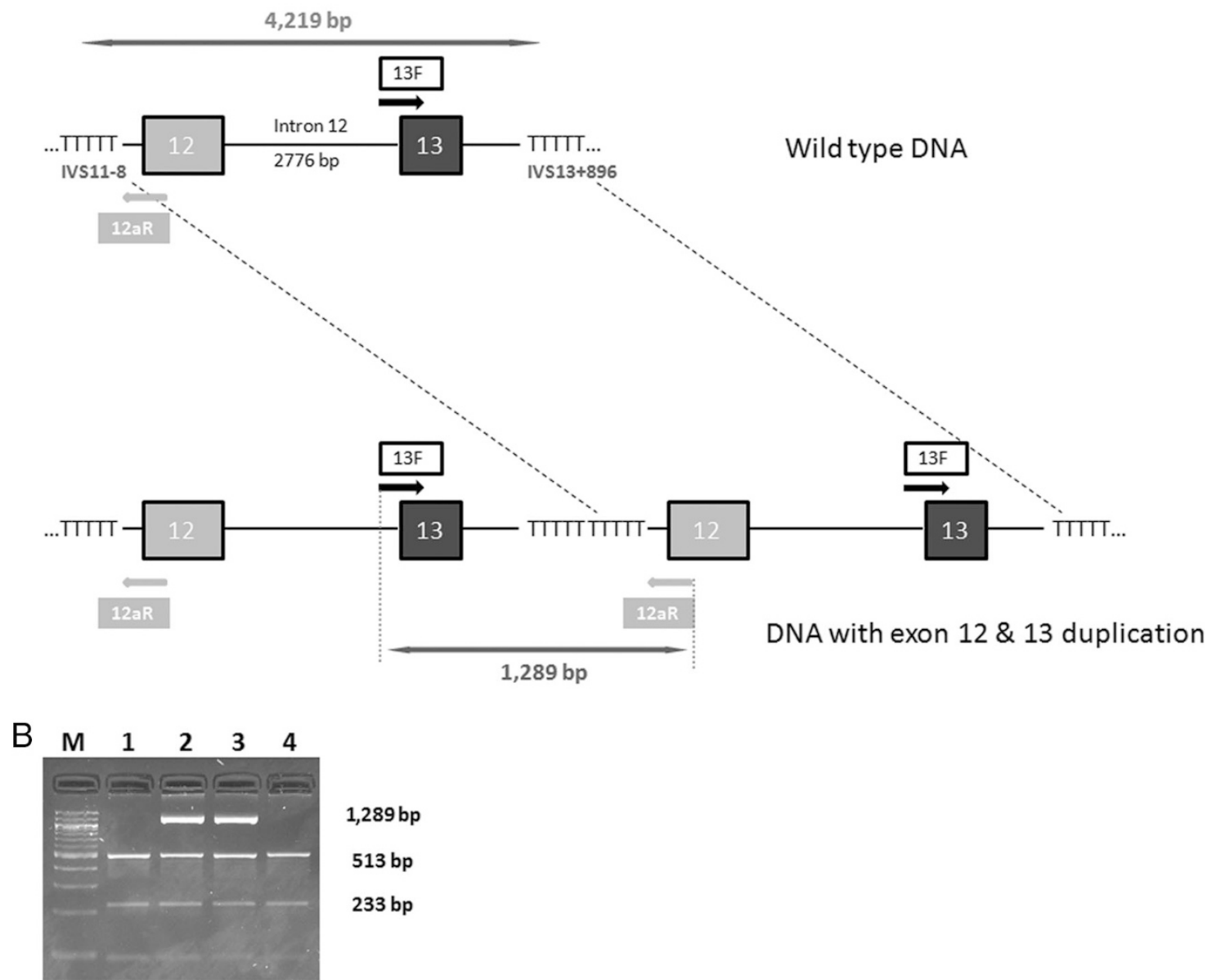

Fig. 3. A, Duplication breakpoints lie in long $T$ stretches, a $(T)_{21}$ lying 8 bp upstream of exon 12 and a $(T)_{19}$ in intron 13 starting $896 \mathrm{bp}$ downstream of exon 13. B, Primers $12 \mathrm{aR}$ and $13 \mathrm{~F}$ were used to screen for the MLH1 duplication in other Colombian CRC families by a rapid specific PCR amplification that resulted in a fragment of $1289 \mathrm{bp}$ in duplication carriers as depicted in lanes 2 and 3. Additional fragments of 233 and 513 bp were detected in all tested samples (lanes 1-4). PCR amplifications were run on TBEX1 in a 3\% agarose gel. bp, base pair; $M, 100$-bp molecular weight marker. 
Finally, we screened for this $M L H 1$ duplication in a CRC cohort to investigate its frequency in that population and explore a putative founder effect in the same population. A total of 123 individuals with $\mathrm{CRC}$ and 80 cancer-free controls were screened. We identified a carrier of this duplication among cases, and none of the controls carried this mutation. The mutation carrier was a woman who presented with ovarian cancer and CRC at the age of 35 and 53 years, respectively. The patient's son and sister also were affected with CRC before age 30 years. Interestingly, both families are independent and from Antioquia, an area in Northwestern Colombia, a population isolate where several founder effects have been detected for other monogenic conditions. ${ }^{12}$

\section{DISCUSSION}

Diagnosis of LS based only on clinical criteria can be difficult because family history information is often lacking, and a typical clinical phenotype for this disease is not present. However, early recognition of LS is essential to identify individuals at high risk who will require intensive cancer surveillance. Therefore, identification of the causative mutation in LS families is extremely useful for genetic counseling and to correctly direct prevention strategies more accurately for mutation carriers at risk of developing $\mathrm{CRC}$ and other malignancies and to avoid excessive clinical intervention in noncarriers.

Molecular studies for LS or other genetic conditions are scarce or nonexistent in some populations. There is only one previous molecular LS study in Colombia. ${ }^{13}$ In that work, 23 families, either fulfilling Amsterdam II criteria (11) or Bethesda guidelines (12) were screened by single strand conformation polymorphism for point mutations in the $M L H 1$ and $M S H 2$ genes, and eight causative mutations were identified, four of them not previously reported. The MSH6 and PMS2 genes were not included in the molecular screening, and neither MLPA nor any other technique able to detect genomic rearrangements was used. The MLH1 duplication identified in our study could not have been detected by the methodology used in that previous study. Indeed, screening for genomic rearrangements in LS is strongly recommended because this type of mutation can be a cause of this hereditary condition as it has been observed in several populations around the world. ${ }^{8-10,14,15}$

The MLH1 duplication (c.1039-8T_1558 + 896Tdup) identified in this study affects exons 12 and 13, and can be considered novel as it has never been reported in LS mutation databases (International Society for Gastrointestinal Hereditary Tumors database, http://www.insight-group.org/mutations/ and Memorial University of Newfoundland Mismatch Repair Genes Variant database, http://www.med.mun.ca/mmrvariants/). ${ }^{16}$ Also, this duplication is most likely not affecting normal splicing of exons 12 and 13 but results in the incorporation of additional exons 12 and 13 into the MLH1 mRNA. This duplication is predicted to create a frameshift and consequently a predicted premature truncation of the translated protein after 44 nucleotides of the duplicated exon 12 (p.520Vfs564X). On the other hand, a premature termination codon early in the coding region of the mRNA will most likely give nonsense-mediated decay and loss of the transcript all together. Without RNA studies of this allele, a solid conclusion about the effect of the duplication cannot be drawn, except that it is most certainly deleterious to the functioning of the allele.

Regarding the mutation's origin at the molecular level, this tandem duplication of $4219 \mathrm{bp}$ is most probably caused by Tstretch-mediated homologous recombination. Duplication breakpoints lie in long $\mathrm{T}$ stretches, a $(\mathrm{T})_{21}$ located $8 \mathrm{bp}$ upstream of exon
12 and a $(\mathrm{T})_{19}$ in intron 13 starting 896 bp downstream of exon 13 (Fig. 3A). Previous reports of LS genomic rearrangements have highlighted the role of Alu-mediated homologous recombination as a common cause for these mutations but not recombination involving $\mathrm{T}$ stretches. ${ }^{14,17-20}$ Also, Alu-mediated homologous recombination seems to be more involved in $\mathrm{MSH} 2$ genomic rearrangements, whereas $M L H 1$ rearrangements may originate from alternative mechanisms. ${ }^{20,21}$

This LS genetic alteration was found in the MLH1 gene, whereas genomic rearrangements in MMR genes have been found more frequently in $M S H 2 .{ }^{22}$ Interestingly, duplications in the MMR genes also seem to be a less frequent mutational event than deletions, and they have been rarely reported. ${ }^{10,22,23}$

Additionally, this novel MLH1 duplication was detected in two independent LS families from the Antioquia area in the Northwestern part of Colombia, and a founder mutation effect may be inferred. A number of this kind of mutations have been identified in the MMR genes, ${ }^{17,24}$ several in the MLH1 gene. ${ }^{25-28}$ Founder mutations of specific populations are important and they can help in the molecular screening of a hereditary disease. Initial screening for these mutations will easily detect carriers, and it would facilitate cumbersome exon-by-exon gene scanning by reducing the number of samples to be screened. The Antioquia area in Colombia is a population isolate where founder effects have been previously detected for other hereditary conditions such as Alzheimer or Parkinson disease. ${ }^{29,30}$ Therefore, it may be recommended to prescreen MLH1-LS Antioquia families for this putative founder mutation before performing the thorough MMR mutational screening.

To conclude, a novel duplication of exons 12 and 13 of the MLH1 gene was detected in two independent families from Colombia as the causative LS mutation. Identifying pathogenic mutations in these families will greatly facilitate presymptomatic diagnoses and genetic counseling, making better therapeutic decisions for carriers before disease manifestation. Also, a putative founder effect for this mutation in the Antioquia area could be inferred with LS molecular screening implications in the Colombian population.

\section{ACKNOWLEDGMENTS}

This work was supported by Grants from the Fondo de Investigación Sanitaria/FEDER (08/0024), Ministerio de Ciencia e Innovación (SAF 07-64873), Asociación Española contra el Cáncer (Fundación Científica y Junta de Barcelona), Fundación de Investigación Médica Mutua Madrileña, and FP7 CHIBCHA Consortium (L.C.-C., M.M.E., I.T., and S.C.-B.). CIBEREHD and CIBERER are funded by the Instituto de Salud Carlos III. V.A.-E. and J.M. are supported by contracts from CIBEREHD, I.M. is supported by a contract from CIBERER, and S.C.-B. is supported by a contract from the Fondo de Investigación Sanitaria (CP 03-0070). F.B. received a research grant from Fundacion Caja Madrid and from Societat Catalana de Digestologia.

\section{REFERENCES}

1. Jasperson KW, Tuohy TM, Neklason DW, Burt RW. Hereditary and familial colon cancer. Gastroenterology 2010;138:2044-2058.

2. Ferlay J, Shin HR, Bray F, Forman D, Mathers C, Parkin DM. Cancer incidence and mortality worldwide: IARC CancerBase No. 10. Lyon, France: International Agency for Research on Cancer, 2010. Available at: http://globocan.iarc.fr. Accessed June 1, 2010.

3. Ángel LA, Giraldo A, Pardo CE. Mortalidad por cánceres del aparato digestivo en Colombia entre 1980 y 1998. Análisis de tendencias y comparación regional. Rev Fac Medicina (Universidad Nacional de Colombia) 2004;52:19-37.

4. Vasen HF, Watson P, Mecklin JP, Lynch HT. New clinical criteria for hered- 
itary nonpolyposis colorectal cancer (HNPCC, Lynch syndrome) proposed by the International Collaborative group on HNPCC. Gastroenterology 1999;116: 1453-1456.

5. Umar A, Boland CR, Terdiman JP, et al. Revised Bethesda Guidelines for hereditary nonpolyposis colorectal cancer (Lynch syndrome) and microsatellite instability. J Natl Cancer Inst 2004;96:261-268.

6. Järvinen HJ, Aarnio M, Mustonen H, et al. Controlled 15-year trial on screening for colorectal cancer in families with hereditary nonpolyposis colorectal cancer. Gastroenterology 2000;118:829-834.

7. Gille JJ, Hogervorst FB, Pals G, et al. Genomic deletions of MSH2 and MLH1 in colorectal cancer families detected by a novel mutation detection approach. Br J Cancer 2002;87:892-897.

8. Wijnen J, van der Klift H, Vasen H, et al. MSH2 genomic deletions are a frequent cause of HNPCC. Nat Genet 1998;20:326-328.

9. Wang Y, Friedl W, Lamberti C, et al. Hereditary nonpolyposis colorectal cancer: frequent occurrence of large genomic deletions in MSH2 and MLH1 genes. Int J Cancer 2003;103:636-641.

10. Castellví-Bel S, Castells A, Strunk M, et al. Genomic rearrangements in MSH2 and MLH1 are rare mutational events in Spanish patients with hereditary nonpolyposis colorectal cancer. Cancer Lett 2005;225:93-98.

11. den Dunnen JT, Antonarakis SE. Mutation nomenclature extensions and suggestions to describe complex mutations: a discussion. Hum Mutat 2000; 15:7-12.

12. Carvajal-Carmona LG, Soto ID, Pineda N, et al. Strong Amerind/white sex bias and a possible Sephardic contribution among the founders of a population in northwest Colombia. Am J Hum Genet 2000;67:1287-1295.

13. Giraldo A, Gómez A, Salguero G, et al. MLH1 and MSH2 mutations in Colombian families with hereditary nonpolyposis colorectal cancer (Lynch syndrome) - description of four novel mutations. Fam Cancer 2005;4:285290.

14. Aissi-Ben Moussa S, Moussa A, Lovecchio T, et al. Identification and characterization of a novel MLH1 genomic rearrangement as the cause of HNPCC in a Tunisian family: evidence for a homologous Alu-mediated recombination. Fam Cancer 2009;8:119-126.

15. Berginc G, Bracko M, Ravnik-Glavac M, Glavac D. Screening for germline mutations of MLH1, MSH2, MSH6 and PMS2 genes in Slovenian colorectal cancer patients: implications for a population specific detection strategy of Lynch syndrome. Fam Cancer 2009;8:421-429.

16. Woods MO, Williams P, Careen A, et al. A new variant database for mismatch repair genes associated with Lynch syndrome. Hum Mutat 2007; 28:669-673.

17. Nyström-Lahti M, Kristo P, Nicolaides NC, et al. Founding mutations and
Alu-mediated recombination in hereditary colon cancer. Nat Med 1995;1: 1203-1206.

18. Mauillon JL, Michel P, Limacher JM, et al. Identification of novel germline hMLH1 mutations including a $22 \mathrm{~kb}$ Alu-mediated deletion in patients with familial colorectal cancer. Cancer Res 1996;56:5728-5733.

19. Kloor M, Sutter C, Wentzensen N, et al. A large MSH2 Alu insertion mutation causes HNPCC in a German kindred. Hum Genet 2004;115:432-438.

20. van der Klift H, Wijnen J, Wagner A, et al. Molecular characterization of the spectrum of genomic deletions in the mismatch repair genes MSH2, MLH1, MSH6, and PMS2 responsible for hereditary nonpolyposis colorectal cancer (HNPCC). Genes Chromosomes Cancer 2005;44:123-138.

21. Viel A, Petronzelli F, Della Puppa L, et al. Different molecular mechanisms underlie genomic deletions in the MLH1 gene. Hum Mutat 2002;20:368374.

22. Charbonnier F, Olschwang S, Wang Q, et al. MSH2 in contrast to MLH1 and MSH6 is frequently inactivated by exonic and promoter rearrangements in hereditary nonpolyposis colorectal cancer. Cancer Res 2002;62:848-853.

23. Baert-Desurmont S, Buisine MP, Bessenay E, et al. Partial duplications of the MSH2 and MLH1 genes in hereditary nonpolyposis colorectal cancer. Eur J Hum Gene 2007;15:383-386.

24. Menéndez M, Castellví-Bel S, Pineda M, et al. Founder effect of a pathogenic MSH2 mutation identified in Spanish families with Lynch syndrome. Clin Genet 2010;78:186-190.

25. Moisio AL, Sistonen P, Weissenbach J, de la Capelle A, Peltomäki P. Age and origin of two common MLH1 mutations predisposing to hereditary colon cancer. Am J Hum Genet 1996;59:1243-1251.

26. Chan TL, Yuen ST, Ho JW, et al. A novel germline 1.8-kb deletion of hMLH1 mimicking alternative splicing: a founder mutation in the Chinese population. Oncogene 2001;20:2976-2981.

27. Caluseriu O, Di Gregorio C, Lucci-Cordisco E, et al. A founder MLH1 mutation in families from the districts of Modena and Reggio-Emilia in northern Italy with hereditary non-polyposis colorectal cancer associated with protein elongation and instability. J Med Genet 2004;41:e34.

28. Chong G, Jarry J, Marcus V, et al. High frequency of exon deletions and putative founder effects in French Canadian Lynch syndrome families. Hum Mutat 2009;30:E797-E812.

29. Lopera F, Ardilla A, Martínez A, et al. Clinical features of early-onset Alzheimer disease in a large kindred with an E280A presenilin-1 mutation. JAMA 1997;277:793-799.

30. Pineda-Trujillo N, Apergi M, Moreno S, et al. A genetic cluster of early onset Parkinson's disease in a Colombian population. Am J Med Genet B Neuropsychiatr Genet 2006;141B:885-889. 